\title{
A Recombinant Humanized Anti-Cocaine Monoclonal Antibody Inhibits the Distribution of Cocaine to the Brain in Rats
}

\author{
Andrew B. Norman, Felicia C. T. Gooden, Michael R. Tabet, and William J. Ball \\ Department of Pharmacology and Cell Biophysics, University of Cincinnati College of Medicine, Cincinnati, Ohio
}

Received January 13, 2014; accepted April 11, 2014

\begin{abstract}
The monoclonal antibody (mAb), h2E2, is a humanized version of the chimeric human/murine anti-cocaine mAb 2E2. The recombinant h2E2 protein was produced in vitro from a transfected mammalian cell line and retained high affinity $(4 \mathrm{nM} \mathrm{K})$ and specificity for cocaine over its inactive metabolites benzoylecgonine (BE) and ecgonine methyl ester. In rats, pharmacokinetic studies of h2E2 $(120 \mathrm{mg} / \mathrm{kg}$ i.v.) showed a long terminal elimination half-life of 9.0 days and a low volume of distribution at steady state $\left(\mathrm{V}_{\mathrm{dss}}\right)$ of $0.3 \mathrm{l} / \mathrm{kg}$. Pretreatment with h2E2 produced a dramatic 8.8-fold increase in the area under the plasma cocaine concentration-time curve (AUC) and in brain a concomitant decrease of $68 \%$ of cocaine's AUC following an i.v. injection of an equimolar cocaine dose. Sequestration of cocaine in
\end{abstract}

plasma by h2E2, shown via reduction of cocaine's $\mathbf{V}_{\mathrm{dss}}$, indicates potential clinical efficacy. Although the binding of cocaine to h2E2 in plasma should inhibit distribution and metabolism, the elimination of cocaine remained multicompartmental and was still rapidly eliminated from plasma despite the presence of h2E2. BE was the major cocaine metabolite, and brain BE concentrations were sixfold higher than in plasma, indicating that cocaine is normally metabolized in the brain. In the presence of h2E2, brain BE concentrations were decreased and plasma BE was increased, consistent with the observed h2E2-induced changes in cocaine disposition. The inhibition of cocaine distribution to the brain confirms the humanized mAb, h2E2, as a lead candidate for development as an immunotherapy for cocaine abuse.
Introduction

Active immunization in humans with cocaine vaccines (Kosten et al., 2002) in some cases produced levels of polyclonal anti-cocaine antibodies that were associated with a decrease in the use of cocaine (Martell et al., 2005), which has demonstrated the potential efficacy of immunotherapy for cocaine abuse. In animals, active immunization with hapten-carrier conjugate vaccines consistently elicits sufficient polyclonal anti-cocaine antibodies to reduce the amount of cocaine entering the brain and concomitantly decrease the behavioral effects of cocaine (Fox et al., 1996). The decrease in brain cocaine concentrations is most likely the mechanism by which the vaccine-induced anti-cocaine antibodies decreased the use of cocaine observed in the clinical studies. Passive immunization with murine anti-cocaine mAbs has also been shown in rats to attenuate the behavioral effects of cocaine (Carrera et al., 1995; Fox et al., 1996; Mets et al., 1998; Carrera et al., 2000) and therefore represents a potential adjunct to active immunization (Kosten and Owens, 2005), or an emergency rescue treatment in instances of cocaine overdose. However, for optimal safety and efficacy in clinical use, anti-cocaine mAbs should have a human sequence and structure (Redwan et al., 2003; Norman and Ball, 2012).

The anti-cocaine monoclonal antibody $(\mathrm{mAb}) 2 \mathrm{E} 2$ was generated by immunization with a hapten-carrier conjugate of a transgenic

This work was supported by the National Institutes of Health National Institute on Drug Abuse [Grant DP1DA031386].

dx.doi.org/10.1124/dmd.114.057034. mouse strain engineered to produce human sequence $\gamma_{1}$ heavy chain (H) and $\kappa$ light chain (L) antibodies (Lonberg, 2005) replacing mouse IgGs. However, the murine $\lambda \mathrm{L}$ chain gene was not knocked out in this transgenic mouse strain, and $2 \mathrm{E} 2$ is a mixed-chain or chimeric $\mathrm{mAb}$ consisting of a human $\gamma_{1} \mathrm{H}$ and murine $\lambda \mathrm{L}$ chain (Norman et al., 2007). This unusual $\mathrm{mAb}$ has a high affinity for cocaine and selectivity for cocaine over its inactive metabolites (Paula et al., 2004), and in vivo studies with mice have demonstrated that infused hybridomaderived 2E2 dramatically increases plasma cocaine levels and decreases the concentration of cocaine reaching the brain (Norman et al., 2007). Furthermore, in rats trained to self-administer cocaine, 2E2 increased the concentration of cocaine required to reinstate this behavior (Norman, et al., 2009). Thus, 2E2, despite being a mixed-chain/chimeric anti-cocaine $\mathrm{mAb}$, had properties that made it a lead candidate for preclinical development.

The $\mathrm{mAb} 2 \mathrm{E} 2$ was obtained from the mAb-producing murine hybridoma cell line grown in nude (severe combined immunodeficiency) mice and then purified from endogenous mouse Igs and serum proteins of the ascites fluid. The mAbs produced from murine-derived hybridoma cell lines cultured in mice are unsuitable for human use due to the potential presence of mouse proteins, endotoxins, and infectious viruses that will compromise safety in humans. Furthermore, the low levels of $2 \mathrm{E} 2$ production and between-batch variations from this in vivo platform meant unacceptably high production costs. In this study, we report, as is typical for therapeutic mAbs, that $2 \mathrm{E} 2$ has been cloned from the murine hybridoma cell line and that constructed genes encoding the $\mathrm{H}$ and $\mathrm{L}$ chains were incorporated into the genome of

ABBREVIATIONS: AUC, concentration-time curve; $\mathrm{AUC}_{(0-\mathrm{t})}$, area under the drug $\mathrm{AUC}$ from time zero to the time of the last data point; $\mathrm{BE}$, benzoylecgonine; $\mathrm{CHO}$, Chinese hamster ovary; ELISA, enzyme-linked immunosorbent assay; EME, ecgonine methyl ester; GC/MS, gas chromatography/mass spectrometry; $\mathrm{H}$, heavy chain; $\mathrm{L}$, light chain; mAb, monoclonal antibody; PBS, phosphate-buffered saline; $\mathrm{t}_{1 / 2 \alpha}$, distribution half-life in a two-compartment pharmacokinetic model; $t_{1 / 2 \beta}$, terminal elimination half-life in a two-compartment pharmacokinetic model; $V_{d s s}$, volume of distribution at steady state. 
Chinese hamster ovary $(\mathrm{CHO})$ cell lines and expressed as a recombinant protein (Bleck, 2012). During the cloning of the $2 \mathrm{E} 2$ human $\mathrm{H}$ chain and the murine $\mathrm{L}$ chain, the murine $\lambda_{\mathrm{c}} \mathrm{L}$ chain constant region was replaced with the $\lambda_{\mathrm{c}}$ human chain constant region, whereas the variable region of the $\lambda$ chain was unmodified. This humanized version of $2 \mathrm{E} 2$, designated h2E2, has been evaluated as our new lead candidate immunotherapeutic agent.

The major metabolites of cocaine in most mammalian species are benzoylecgonine (BE) and ecgonine methyl ester (EME), formed by enzymatic hydrolysis of cocaine's methyl-ester and benzoyl-ester moieties, respectively (Warner and Norman, 2000). Therefore, it is critical for a therapeutic antibody to have lower affinity for the inactive metabolites of cocaine as these tend to have longer half-lives than cocaine and can build up to higher concentrations that may compete for cocaine binding after multiple doses of cocaine are consumed. Although the mAb 2E2 has selectivity for cocaine over its inactive metabolites, it does have an appreciable affinity for BE as compared with the much lower affinity for EME and ecgonine. Therefore, we investigated whether h2E2 retained 2E2's specificity for cocaine over its inactive metabolites. Previous measurements of the effect of 2E2 on the disposition of cocaine were in mice in which the major metabolite is EME. In contrast, in rats the major metabolite is BE. The efficacy of h2E2 as measured by the effects on the disposition of cocaine was therefore tested in rats. We report in this work our demonstration that the recombinant anti-cocaine $\mathrm{mAb}, \mathrm{h} 2 \mathrm{E} 2$, retains the same high affinity and specificity for cocaine over its major inactive metabolites and significantly decreases the distribution of cocaine to the brain in rats.

\section{Materials and Methods}

The Generation and Production of h2E2. In this study, we report the generation of stably transfected CHO-S cells that have incorporated multiple gene copies of the human sequence $\gamma_{1} \mathrm{H}$ chain of mAb 2E2 and a partially humanized version of its murine $\lambda \mathrm{L}$ chain. This humanized version of 2E2, designated h2E2, has been transfected into CHO-S cells, and selected cell lines with high levels of expression of the recombinant h2E2 have been isolated. Cultured in serum-free medium, these cell lines secrete the recombinant $\mathrm{mAb}$ $\mathrm{h} 2 \mathrm{E} 2$ at concentrations approaching $1 \mathrm{~g} / \mathrm{l}$, and h2E2 is readily purified by protein A affinity chromatography with initial yields of $0.5 \mathrm{~g} / \mathrm{l}$. An initial 10-1 production run yielded $5.7 \mathrm{~g}$ purified $\mathrm{h} 2 \mathrm{E} 2$. This is the first time that sufficient quantities of a humanized anti-cocaine mAb have been available to allow for studies in animals larger than mice.

The partial amino acid sequences of the $\mathrm{H}$ and $\mathrm{L}$ chains of mAb 2E2 produced from the hybridoma cell line were initially determined by liquid chromatography/tandem mass spectroscopy analysis of their trypsin-digested fragments. In addition, the predicted amino acid sequences of the $\mathrm{H}$ and $\mathrm{L}$ chains of $2 \mathrm{E} 2$ were obtained from the cDNAs generated from the full-length $\mathrm{H}$ and L chain mRNAs (Paula et al., 2004). The proprietary GPEx gene expression technology (Bleck, 2012) of Catalent (Madison, WI) was used to carry out transfections that generated stably transfected CHO-S cells containing multiple transgene copies inserted into transcriptionally active regions of the genome. The GPEx technology utilizes replication-defective retroviral vectors derived from Moloney murine leukemia virus to achieve the targeting and the insertion of the RNA-coded genes for the h2E2 $\gamma_{1} \mathrm{H}$ and the modified $\lambda \mathrm{L}$ chain. The electronic DNA and protein $\mathrm{H}$ and $\mathrm{L}$ sequences for $2 \mathrm{E} 2$ as well as encoding plasmids were used to construct the retroviral vectors. The reengineering of the murine $\lambda \mathrm{L}$ chain of the chimeric 2E2 was accomplished using standard molecular biology techniques to synthetically construct the $\mathrm{L}$ chain with the human $\lambda_{\mathrm{c}}$ constant regions sequence replacing the murine sequence while leaving the $\lambda_{\mathrm{v}}$ region unmodified. The construction of the new expression constructs was verified by DNA sequencing.

After five transfection rounds of viral infection with the $\mathrm{H}$ chain and four rounds with the $\mathrm{L}$ chain containing retrovectors, the identification by dilution cloning of 24 cell lines (average gene copy numbers/clone: $\mathrm{H}$ chain, 1.75; L chain, 4.5) with high mAb production levels was achieved without antibioticor methotrexate-dependent cell selection procedures. The expressed and secreted recombinant $\mathrm{h} 2 \mathrm{E} 2 \mathrm{mAb}$ used for these studies was affinity purified from the serum-free culture media (PF CHO LS media) of a batch culture growth of the selected top five stably transfected CHO-S cloned lines by protein A high-performance liquid chromatography. The final concentration of h2E2 in phosphate-buffered saline (PBS) was $5 \mathrm{mg} / \mathrm{ml}$. The amino acid sequences of the $\mathrm{H}$ and $\mathrm{L}$ chains of the purified h2E2 were confirmed by liquid chromatography/mass spectrometry analysis of the fragments from the trypsin digestion of $\mathrm{H}$ and $\mathrm{L}$ chains following separation via SDS-gel electrophoresis. Approximately $94 \%$ of the residue sequences were identified, and all of these were consistent with those predicted from the DNA sequences.

$\left[{ }^{3} \mathbf{H}\right]$ Cocaine-Binding Studies. The binding affinities of $2 \mathrm{E} 2$ and recombinant h2E2 for cocaine were measured by the equilibrium binding to $\left[{ }^{3} \mathrm{H}\right]$ cocaine in PBS ( $\left.\mathrm{pH} 7.0\right)$ at room temperature and incubated for 6 hours before separation of bound and free radioligand. The separation was accomplished by ultrafiltration of the precipitated anti-human $\mathrm{Fc}$ region antibody-h2E2 or 2E2cocaine complex through glass fiber (GF/B) filters (GE Healthcare, Fairfield, CT). The $K_{d}$ of $\left[{ }^{3} \mathrm{H}\right]$ cocaine for the binding to mAbs $2 \mathrm{E} 2$ and h2E2 was calculated from best fit of a hyperbolic function to the specific binding as a function of $\left[{ }^{3} \mathrm{H}\right]$ cocaine concentration.

Specificity of h2E2 for Cocaine. The relative binding affinities of cocaine and its major metabolites for h2E2 used a competition enzyme-linked immunosorbent assay (ELISA). The wells in the polyvinyl chloride plates were coated with the hapten BE coupled to 1,4-diaminobutane (putrescene)derivatized bovine serum albumin. The ligand recognition site of h2E2 was generated in response to a hapten-cationized keyhole limpet hemocyanin conjugate that has the amide, four carbon-linker derivative of $\mathrm{BE}$ as the hapten. Consequently, the mAb has a higher affinity for the ethyl and propyl ester derivatives of cocaine than for cocaine, but a lower affinity for BE. The binding of h2E2 to the conjugate was measured in the presence of a range of concentrations of cocaine or various metabolites. Hapten-bound h2E2 was measured using a biotinylated purified goat anti-human IgG polyclonal antibody described previously (Paula et al., 2004). The relative binding affinities of the metabolites were determined by the ratios of their $\mathrm{IC}_{50}$ values to the $\mathrm{IC}_{50}$ value for cocaine.

Animals. Jugular vein-catheterized male Sprague-Dawley rats (250 g) were purchased from Harlan (Indianapolis, IN). Animals were housed individually with free access to food and water and kept on a 12-hour light/dark cycle. Studies using animals were carried out in accordance with the Guide for the Care and Use of Laboratory Animals under a protocol approved by the Institutional Animal Care and Use Committee at the College of Medicine, University of Cincinnati.

mAb h2E2 Pharmacokinetic Studies. The in vivo concentrations of h2E2 following a single injection (120 mg/kg i.v.) were determined using an ELISA described previously (Paula et al., 2004; Norman et al., 2007) that compared the quantity of $\mathrm{mAb}$ in varying dilutions of the rat blood samples with that quantified in a standard curve using known dilutions of purified h2E2. The blood samples ( $10 \mu 1$ each) were collected from a small incision at the tip of the tail, and the samples were collected at various time points up to 7 weeks after the administration of h2E2.

Cocaine Pharmacokinetic Studies in Rat Plasma. The antibody $(120 \mathrm{mg} / \mathrm{kg}$, which has equimolar cocaine binding sites with $0.56 \mathrm{mg} / \mathrm{kg}$ cocaine as the $\mathrm{HCl}$ salt) in PBS or an equivalent volume of vehicle (PBS) was infused i.v. at a rate of approximately $0.35 \mathrm{ml} / \mathrm{min}$ for up to 2 minutes, with the animal held under mild restraint. One hour after completion of the infusion of $\mathrm{mAb}$, cocaine $\mathrm{HCl}$ $(0.56 \mathrm{mg} / \mathrm{kg})$ plus heparin $(400 \mathrm{U} / \mathrm{kg})$ was injected i.v. through the same catheter at a volume of $4.0 \mathrm{ml} / \mathrm{kg}$ body weight. At most sampling times, sodium pentobarbital $(45 \mathrm{mg} / \mathrm{kg}$, i.p.) was administered three minutes prior to sacrificing the animal. For the 0.75 and 1.5 -minute time point, the cocaine was injected into anesthetized rats. At the designated times after the injection of cocaine, anesthetized rats were sacrificed by decapitation, and trunk blood (typically 3-5 ml) was collected. From this volume, two 1-ml aliquots were retained and placed in polypropylene microcentrifuge tubes containing $11.2 \mu \mathrm{l}$ heparin $(1.0 \mathrm{U} / \mu \mathrm{l})$ to inhibit blood coagulation and $\mathrm{NaF}(16 \mathrm{mg} / 0.8 \mathrm{ml}$ blood $)$ to inhibit enzymatic hydrolysis of cocaine (Warner and Norman, 2000). The blood samples were centrifuged at $5000 \mathrm{~g}$ for 3 minutes, and then the plasma (typically $0.4-0.8 \mathrm{ml}$ ) was carefully separated from packed red blood cells, 
placed into sterile 1.5-ml Eppendorf microcentrifuge tubes, rapidly frozen on dry ice, and stored at $-80^{\circ} \mathrm{C}$ until analysis.

Cocaine Pharmacokinetics in Rat Brain. The whole brain was quickly removed from each decapitated animal, surface blood was blotted away, and the brain was placed in a polypropylene tube, rapidly frozen on dry ice, and stored at $-80^{\circ} \mathrm{C}$ until analysis. For analysis, individual brains were weighed and cold deionized, distilled water was added to produce a total volume of $1 \mathrm{ml}$, and then the solution was homogenized and centrifuged at 13,000 rpm for 45 minutes at $4^{\circ} \mathrm{C}$. The resulting supernatants $(0.4-0.6 \mathrm{ml})$ were collected into sterile polypropylene microcentrifuge tubes, and an aliquot $(0.05-0.40 \mathrm{ml})$ was processed for cocaine/metabolite analysis by gas chromatography/mass spectrometry $(\mathrm{GC} / \mathrm{MS})$ and hemoglobin content. Any remaining sample was stored at $-80^{\circ} \mathrm{C}$.

Determination of Blood and Brain Hemoglobin Concentrations. At the same time as plasma sample collections, a separate sample of blood (approximately $100 \mu \mathrm{l}$ ) was collected from each animal and rapidly frozen on dry ice before storage at $-80^{\circ} \mathrm{C}$. The concentration of hemoglobin and, where appropriate, h2E2 was measured in these samples.

The hemoglobin contents of brain and blood were quantified spectroscopically by combining the method reported by Choudhri et al. (1997) and a protocol provided by Pointe Scientific (Canton, MI). This procedure was identical to that reported in a previous study (Norman et al., 2007). The mean \pm S.E.M. concentration of hemoglobin in whole blood and brain was $7.22 \pm 0.46$ $\mathrm{g} / \mathrm{dl}$ and $0.18 \pm 0.05 \mathrm{~g} / \mathrm{dl}$, respectively. The average hemoglobin content in brain tissue relative to that present in whole blood was, therefore, $2.5 \%$.

Solid-Phase Extraction and Quantification of Cocaine and Metabolites from Plasma and Brain. Cocaine, BE, and EME concentrations in plasma and brain were measured using solid-phase extraction, followed by GC/MS, according to the procedures detailed by Norman et al. (2007).

Chemicals, Reagents, and Reference Standards. $\left[{ }^{3} \mathrm{H}\right]$ Cocaine $(26 \mathrm{Ci} / \mathrm{mmol})$ was purchased from Perkin-Elmer NEN Radiochemical (Boston, MA). Standard solutions of cocaine, BE, and EME (each $1 \mathrm{mg} / \mathrm{ml}$ ) were prepared in methanol or acetonitrile and served as stock solutions for preparing the reference standard curves for GC/MS quantification. The deuterium-labeled cocaine- $\mathrm{D}_{3}, \mathrm{BE}-\mathrm{D}_{3}$, and EME- $\mathrm{D}_{3}$ that were used as the internal standards $(0.1 \mathrm{mg} / \mathrm{ml}$ each in methanol or acetonitrile) and the derivatizing reagent N,O-bis (trimethylsilyl)trifluoroacetamide with $1 \%$ trimethylchlorosilane were purchased from Cerilliant (Round Rock, TX). The high and low hemoglobin concentration standards were purchased from Pointe Scientific. All other chemicals and immunoreagents were purchased from Sigma-Aldrich (St. Louis, MO) or Pierce Chemicals (Rockford, IL). All reagents and organic solvents were of analytical or high-performance liquid chromatography grade.

Data Analysis and Statistics. Cocaine and h2E2 pharmacokinetic data and the h2E2 and 2E2 pharmacodynamic (radioligand-binding) data were analyzed using the program PKSolver (Department of Pharmaceutics, China Pharmaceutical University, Nanjing, China). PKSolver (Zhang et al., 2010) is a Microsoft Excel add-in program that has been validated for pharmacokinetic/ pharmacodynamic data analysis against WinNonLin (Certara USA, St. Louis, MO). The program provides Akaike Information Criterion and Schwartz Bayesian Criterion measures of model diagnostics of the data to the pharmacokinetic models that were used. Pharmacokinetic data were analyzed according to a two-compartment model based on previous evidence that a singlecompartment model gave a poor fit to the cocaine pharmacokinetic data (Norman et al., 2007). The two-compartment pharmacokinetic model used to fit the data assumed that cocaine distributed between a central and a peripheral compartment. The fit to the data using a two-compartment model yielded optimal Akaike Information Criterion and Schwartz Bayesian Criterion measures, lack of systematic deviation from the data points, and a concomitant reduction in the sum of squares residuals. Applying pharmacokinetic models that assumed that cocaine distributed between more than two compartments only slightly improved the fit to the observed data, and this additional complexity was considered unnecessary.

The h2E2 and 2E2 data from $\left[{ }^{3} \mathrm{H}\right]$ cocaine-binding studies were analyzed in PKSolver to calculate the $K_{d}$ through the best fit of a hyperbolic function to the specific binding as a function of $\left[{ }^{3} \mathrm{H}\right]$ cocaine concentration.

The formation of metabolites was analyzed using SigmaPlot (Systat Software, San Jose, CA) global curve fit analyses. Because the formation of metabolites continued over the time course of the experiments and the elimination of the major metabolite, BE, extended beyond the time course of the study, a pharmacokinetic analysis of $\mathrm{BE}$ was deemed inappropriate.

\section{Results}

Binding Properties of Recombinant mAb h2E2. As shown in Figure 1, the re-engineered $\mathrm{mAb} h 2 \mathrm{E} 2\left(\mathrm{~K}_{\mathrm{d}}=3.9 \mathrm{nM}\right)$ retained a high affinity for $\left[{ }^{3} \mathrm{H}\right]$ cocaine similar to that of the mouse hybridoma cellderived $2 \mathrm{E} 2\left(\mathrm{~K}_{\mathrm{d}}=4.4 \mathrm{nM}\right)$ with the cocaine affinities of the two structural variants indistinguishable. In addition, h2E2 retained essentially the same specificity as $2 \mathrm{E} 2$ for cocaine over its major inactive metabolites, BE, EME, and ecgonine (Table 1). Furthermore, like 2E2, h2E2 had a higher affinity for the active metabolite cocaethylene than for cocaine and a moderately high affinity for the active metabolite norcocaine (Table 1).

The Pharmacokinetics of mAb h2E2. To determine the pharmacokinetics of $\mathrm{mAb}$ h2E2 in rats $(n=4)$, the first samples of tail vein blood were taken 1 hour after completion of the i.v. infusion of h2E2 $(120 \mathrm{mg} / \mathrm{kg})$ via the implanted jugular vein catheter. The initial mean blood concentration of mAb was determined to be $600 \mu \mathrm{g} / \mathrm{ml}$. As shown in Figure 2, the observed mean blood concentrations of h2E2 were highest at the earliest time points. There was an initial rapid decrease in h2E2 concentrations over the first 2 days, followed by a slower exponential decrease over the 6-week duration of the study. The declining concentrations of h2E2 in blood were adequately described by a two-compartment pharmacokinetic model with a distribution half-life $\left(\mathrm{t}_{1 / 2 \alpha}\right)$ of 19 hours and an elimination half-life $\left(\mathrm{t}_{1 / 2 \beta}\right)$ of 9.0 days (Fig. 2). This model gave a calculated volume of distribution at steady state $\left(\mathrm{V}_{\mathrm{dss}}\right)$ for $\mathrm{h} 2 \mathrm{E} 2$ of $0.31 / \mathrm{kg}$.

The Pharmacokinetics of Cocaine in Plasma and Brain. The pharmacokinetics of cocaine in rat plasma subsequent to its i.v. injection via the jugular vein was determined. The highest mean plasma concentration measured $(380 \mathrm{ng} / \mathrm{ml})$ was observed at the earliest sampling time, after which a rapid decline in cocaine concentrations was observed (Fig. 3A). The pharmacokinetic model generated parameter estimates for the cocaine $t_{1 / 2 \alpha}$ and $t_{1 / 2 \beta}$ of 0.4 and 32.9 minutes, respectively. The calculated cocaine $\mathrm{V}_{\mathrm{dss}}$ was $2.61 / \mathrm{kg}$.

As shown in Figure 3B, the cocaine concentration-time profile in rat brain differed substantially from that observed in the plasma (Fig. 3A).

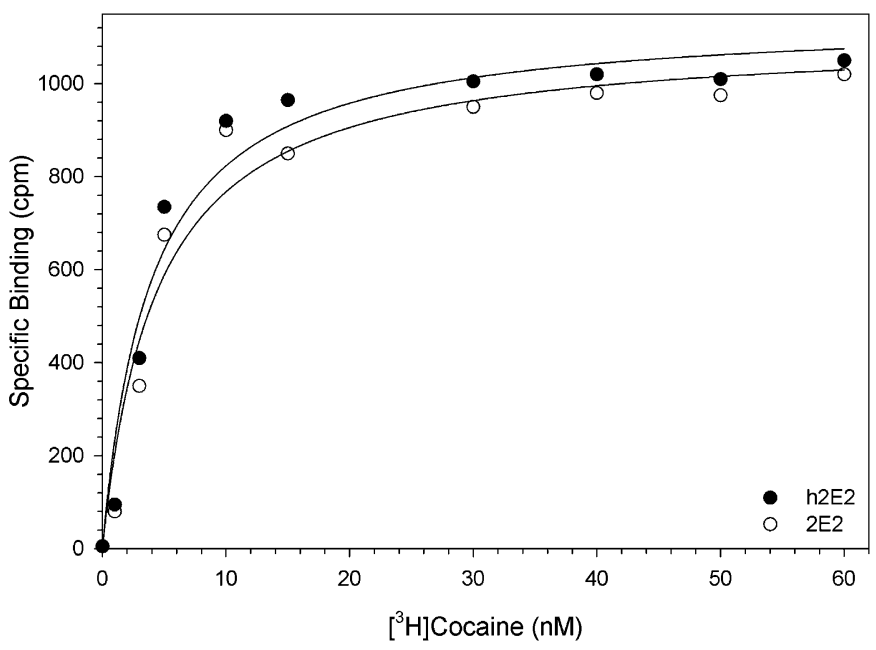

Fig. 1. The binding affinities of $2 \mathrm{E} 2$ and $\mathrm{h} 2 \mathrm{E} 2$ for $\left[{ }^{3} \mathrm{H}\right]$ cocaine. Antibodies and $\left.{ }^{3} \mathrm{H}\right]$ cocaine were incubated in PBS at $\mathrm{pH} 7.0$ at room temperature for 6 hours. The $\mathrm{K}_{\mathrm{d}}$, defined as the concentration of $\left[{ }^{3} \mathrm{H}\right]$ cocaine at which $50 \%$ of $\mathrm{B}_{\max }$ occurred, was $4.4 \mathrm{nM}$ for $2 \mathrm{E} 2$ and $3.9 \mathrm{nM}$ for h2E2. 
TABLE 1

The relative binding affinities of monoclonal antibodies $2 \mathrm{E} 2$ and $\mathrm{h} 2 \mathrm{E} 2$ for cocaine and its metabolites

The relative binding affinities (RBAs) were measured using a competition enzyme-linked immunosorbent assay, as described in Materials and Methods. The measured $\mathrm{IC}_{50}$ values for each metabolite were compared with that of cocaine, which was designated a RBA of 1 . Values higher or lower than 1 indicate, respectively, a lower or higher affinity for h2E2 than that of cocaine.

\begin{tabular}{lcc}
\hline & \multicolumn{2}{c}{ Relative Binding Affinity } \\
\cline { 2 - 3 } \multicolumn{1}{c}{ Metabolite } & $2 \mathrm{E} 2$ & $\mathrm{~h} 2 \mathrm{E} 2$ \\
\hline Cocaine & 1 & 1 \\
Cocaethylene & 0.5 & 0.6 \\
Norcocaine & 7 & 8 \\
Benzoylecgonine & 11 & 7 \\
Ecgonine methyl ester & 1800 & 1900 \\
Ecgonine & 23,000 & 18,000 \\
\hline
\end{tabular}

The initial mean brain cocaine concentrations (corrected for cocaine present in residual blood) at 45 seconds (1500 ng/g) after the injection were approximately fourfold higher than that measured in the plasma at that time. The brain cocaine concentrations subsequently increased further, with the highest mean measured concentrations observed at 1.5 minutes $(1800 \mathrm{ng} / \mathrm{g})$, after which cocaine concentrations rapidly declined. A pharmacokinetic model that assumed a first-order cocaine input to the brain and a first-order elimination was used to describe the increase and subsequent decrease in brain cocaine concentrations. The estimated input half-life in a noncompartmental pharmacokinetic model was 0.7 minute, and the elimination half-life in a noncompartmental pharmacokinetic model was 25 minutes. The estimated area under the drug concentration-time curve (AUC) from time zero to the time of the last data point $\left[\mathrm{AUC}_{(0-\mathrm{t})}\right]$ for brain cocaine concentrations was $29,900(\mathrm{ng} / \mathrm{g}) \times$ minutes.

Effect of h2E2 on the Pharmacokinetics of Cocaine in Plasma and Brain. As shown in Figure 3A, in the presence of h2E2, the peak mean plasma concentrations $(6300 \mathrm{ng} / \mathrm{ml})$ of cocaine were also observed at the earliest time point ( 45 seconds) after h2E2 injection. This is similar to what was observed in the absence of h2E2. However, the

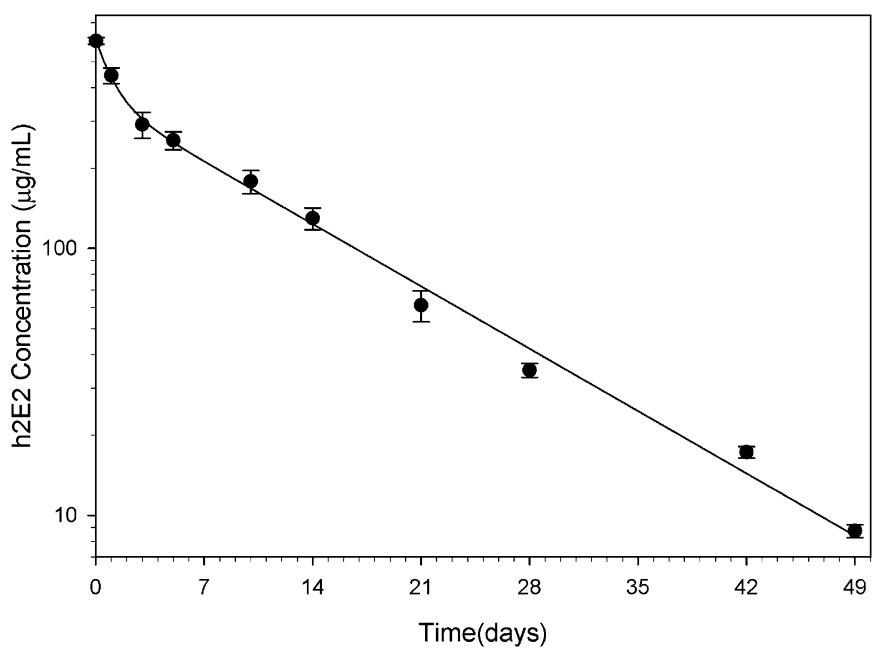

Fig. 2. The pharmacokinetics of the anti-cocaine $\mathrm{mAb} h 2 \mathrm{E} 2$ in rats. Animals received an i.v. infusion of $120 \mathrm{mg} / \mathrm{kg}$ h2E2. Samples of blood $(10 \mu \mathrm{l})$ were obtained from tail veins at the indicated times after completion of the mAb infusion. Concentrations of h2E2 in blood were determined using an ELISA. Data points represent the mean \pm S.E.M. from four rats. The $V_{\mathrm{dss}}$ was $0.31 / \mathrm{kg}$. A twocompartment model with a $t_{1 / 2 \alpha}$ of 19 hours described the distribution phase, and a $t_{1 / 2 \beta}$ of 9.0 days described the elimination phase, represented by the best-fit regression line.
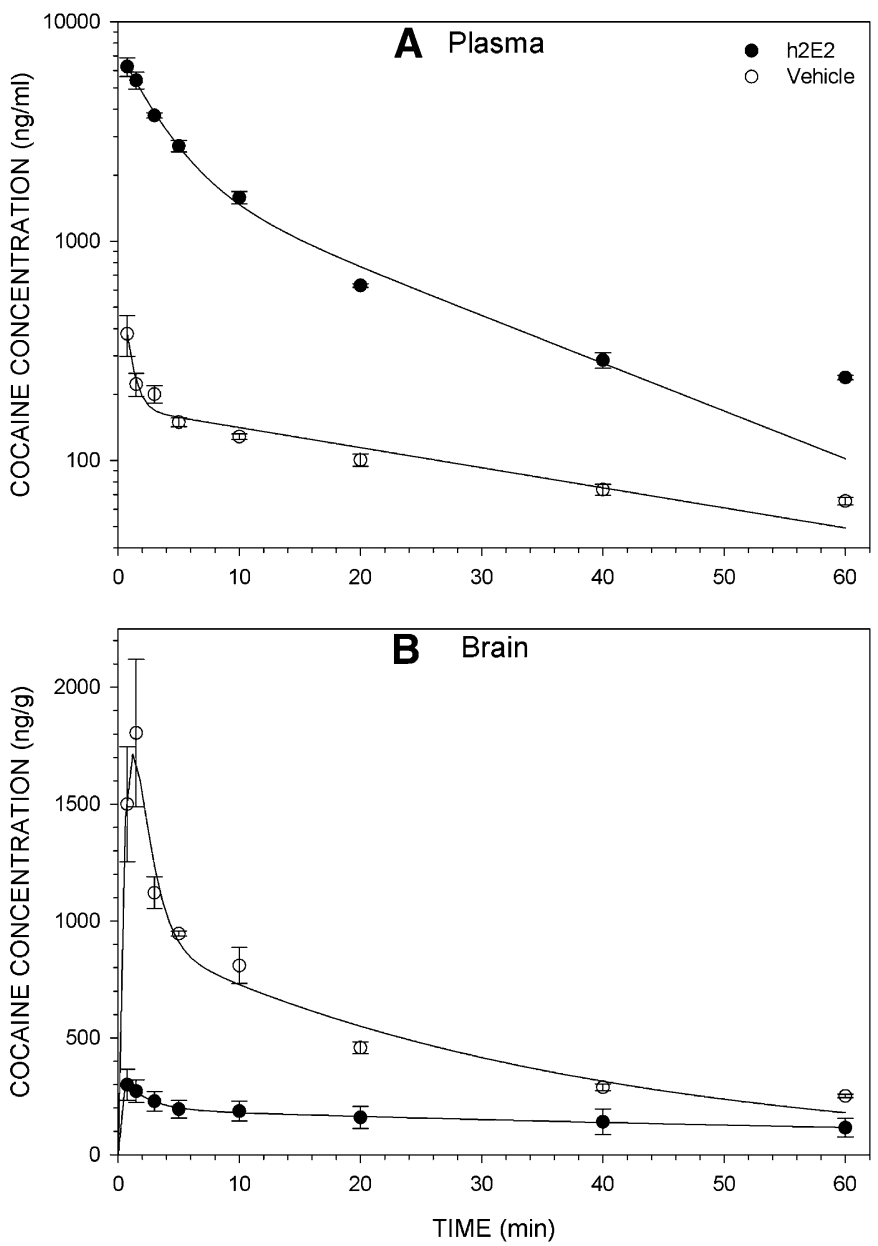

Fig. 3. The effect of h2E 2 on the pharmacokinetics of cocaine in plasma (A) and brain (B) in rats. Rats received an i.v. infusion of $120 \mathrm{mg} / \mathrm{kg} \mathrm{h} 2 \mathrm{E} 2$. One hour later the rats received an i.v. injection of cocaine $\mathrm{HCl}(0.56 \mathrm{mg} / \mathrm{kg})$. The animals were sacrificed at the indicated times, and blood and the brain were collected. Cocaine concentrations were determined by GC/MS. Each data point represents the mean \pm S.E.M. from three rats. In the absence of h2E2 (open circles), the cocaine concentration-time profile in plasma (A) was described by a two-compartment pharmacokinetic model with a $t_{1 / 2 \alpha}$ of 0.4 minute and a $t_{1 / 2 \beta}$ of 32.9 minutes. In the presence of h2E2 (closed circles), a two-compartment pharmacokinetic model indicated a $t_{1 / 2 \alpha}$ of 2.2 minutes and a $t_{1 / 2 \beta}$ of 13.8 minutes. h2E2 produced a 8.8 -fold increase in the area under the plasma cocaine AUC. The $\mathrm{V}_{\mathrm{dss}}$ in the absence and presence of h2E2 was 2.6 and $0.1 \mathrm{l} / \mathrm{kg}$, respectively. In the brain (B) in the absence of h2E2 (open circles), a two-compartment pharmacokinetic model with an AUC of $29,900(\mathrm{ng} / \mathrm{g}) \times$ minutes described the cocaine concentration-time profile. In the presence of h2E2 (closed circles), a two-compartment pharmacokinetic model with an AUC of $9460(\mathrm{ng} / \mathrm{g}) \times$ minutes described the cocaine concentration-time profile. h2E 2 produced a $68 \%$ decrease in the brain cocaine AUC.

peak plasma concentration in the presence of h2E2 was 16.5 -fold higher. Furthermore, the biexponential decrease in cocaine concentrations observed in the absence of h2E2 held true in the presence of $\mathrm{h} 2 \mathrm{E} 2$ as well. The distribution and elimination phases were described by a $t_{1 / 2 \alpha}$ of 2.2 minutes and a $t_{1 / 2 \beta}$ of 13.8 minutes (Table 2). h2E2 also produced a sustained increase in the plasma cocaine concentration that resulted in an 8.8-fold increase in the plasma AUC (Table 2). Consistent with these results, the calculated $\mathrm{V}_{\mathrm{dss}}$ of cocaine in the presence of h2E2 was $0.1 \mathrm{l} / \mathrm{kg}$ compared with $2.61 / \mathrm{kg}$ in the absence of h2E2 (Table 2). The calculated plasma clearance of cocaine was dramatically reduced by $97 \%$ in the presence of h2E2 (Table 2).

In the presence of h2E2, the highest mean brain cocaine concentrations $(450 \mathrm{ng} / \mathrm{g})$ were observed at the earliest sample times with a subsequent decline over time (Fig. 3B). Importantly, h2E2 abolished 
TABLE 2

Pharmacokinetic parameter estimates for cocaine in the presence and absence of h2E2

The parameter estimates were generated using the program PKSolver from the models shown in Figure 3, A and B. +, Represents the fold increase from the control values; -, represents the percent decrease from control values.

\begin{tabular}{|c|c|c|c|c|c|c|}
\hline \multirow{2}{*}{ Parameters } & \multicolumn{3}{|c|}{ Plasma } & \multicolumn{3}{|c|}{ Brain } \\
\hline & Control & h2E2 & Change & Control & h2E2 & Change \\
\hline $\operatorname{AUC}_{(0-\mathrm{t})}[(\mathrm{ng} / \mathrm{ml})$ or $(\mathrm{ng} / \mathrm{g})] \times$ minutes & 6370 & 56,200 & +8.8 -fold & 29,900 & 9460 & $-68 \%$ \\
\hline $\operatorname{AUC}_{(0-\infty)}[(\mathrm{ng} / \mathrm{ml})$ or $(\mathrm{ng} / \mathrm{g})] \times$ minutes & 8710 & 58,200 & +6.7 -fold & 36,300 & 23,100 & $-36 \%$ \\
\hline $\mathrm{t}_{1 / 2 \alpha}$ (minutes) & 0.4 & 2.2 & +5.5 -fold & 0.7 & 1.5 & +2.1 -fold \\
\hline $\mathrm{t}_{1 / 2 \beta}$ (minutes) & 32.9 & 13.8 & $-58 \%$ & 24.8 & 81.2 & +3.3 -fold \\
\hline $\mathrm{Cl}(1 / \mathrm{min})$ & 0.7 & 0.02 & $-97 \%$ & 0.1 & 0.3 & +3.0 -fold \\
\hline $\mathrm{V}_{\mathrm{dss}}(1 / \mathrm{kg})$ & 2.6 & 0.1 & $-96 \%$ & 0.5 & 2.5 & +5.0 -fold \\
\hline
\end{tabular}

$\mathrm{AUC}_{(0-\mathrm{t})}$, area under the drug concentration-time curve from time zero to the time of the last data point; $\mathrm{AUC}_{(0-\infty)}$, area under the drug concentration-time curve from time zero extrapolated to infinity; $\mathrm{Cl}$, plasma clearance; $\mathrm{t}_{1 / 2 \alpha}$, distribution half-life in a two-compartmen pharmacokinetic model; $\mathrm{t}_{1 / 2 \beta}$, terminal elimination half-life in a two-compartment pharmacokinetic model; $\mathrm{V}_{\mathrm{dss}}$, volume of distribution at steady state.

the initial fast increase and peaking of the cocaine concentrations. In the presence of h2E2, the estimated cocaine $\mathrm{AUC}_{(0-\mathrm{t})}$ was $9460(\mathrm{ng} / \mathrm{g}) \times$ minutes, which represents an approximately $68 \%$ decrease from the AUC in the absence of h2E2 (Fig. 3B; Table 2).

The Metabolism of Cocaine and the Effect of h2E2. Concomitant with the determination of cocaine concentrations in the plasma and brain of rats, the concentrations of the inactive metabolites of cocaine, $\mathrm{BE}$ and EME, were measured. The major metabolite in both plasma and brain was BE, with EME detectable only in trace amounts. A maximum EME concentration of $9 \mathrm{ng} / \mathrm{ml}$ was detected in plasma, and a higher concentration of $29 \mathrm{ng} / \mathrm{g}$ was detected in brain. These values were about $88 \%$ lower and $93 \%$ lower than the peak BE concentrations in plasma and brain, respectively. Therefore, the metabolism of cocaine to EME was considered negligible, and EME concentration data are not shown. As shown in Figure 4A, in the absence of h2E2, $\mathrm{BE}$ concentrations increased gradually in plasma, reaching a peak of $73 \mathrm{ng} / \mathrm{ml}$ at the last time point measured. BE was also found in the brain with a mean peak concentration of $430 \mathrm{ng} / \mathrm{g}$ at approximately 5 minutes and then declined gradually. Thus, the pharmacokinetics of $\mathrm{BE}$ were different in plasma and brain, and the peak brain BE concentration was approximately sixfold higher in brain than in plasma. In the presence of $\mathrm{h} 2 \mathrm{E} 2$, the time course of $\mathrm{BE}$ formation in the plasma was similar to that observed in the absence of h2E2, but the mean peak $\mathrm{BE}$ concentration in plasma was increased approximately twofold (Fig. 4A). In the brain in the presence of h2E2, the highest BE concentration occurred at the earliest time point measured before declining to a stable concentration. Although initial BE concentrations were similar whether h2E2 was present or absent, only in the absence of antibody did BE concentrations increase to a peak before declining (Fig. 4B). Unfortunately, the limited time course of these studies compromised the ability to more thoroughly estimate the pharmacokinetic parameters for BE.

\section{Discussion}

The recombinant humanized $\mathrm{mAb}, \mathrm{h} 2 \mathrm{E} 2$, retained the same high affinity and specificity for cocaine over cocaine's major inactive metabolites, BE, EME, and ecgonine, as the hybridoma-derived chimeric human/murine mAb 2E2 (Paula et al., 2004). Thus, exchanging the murine $\lambda$ constant region for a human $\lambda$ sequence did not adversely alter the binding characteristics of the anti-cocaine $\mathrm{mAb}$.

The pharmacokinetics of h2E2 and the effects of h2E2 on cocaine's pharmacokinetics in rats were then investigated. The $\mathrm{V}_{\mathrm{dss}}$ of h2E2 in rats was similar to that observed in mice using 2E2 (Norman et al., 2007) and to that previously reported for human polyclonal IgG1 antibodies in rats (Bazin-Redureau et al., 1997). This low $\mathrm{V}_{\mathrm{dss}}$ is consistent with h2E2's distribution being predominantly restricted to the blood and interstitial fluid volumes. Additionally, the elimination
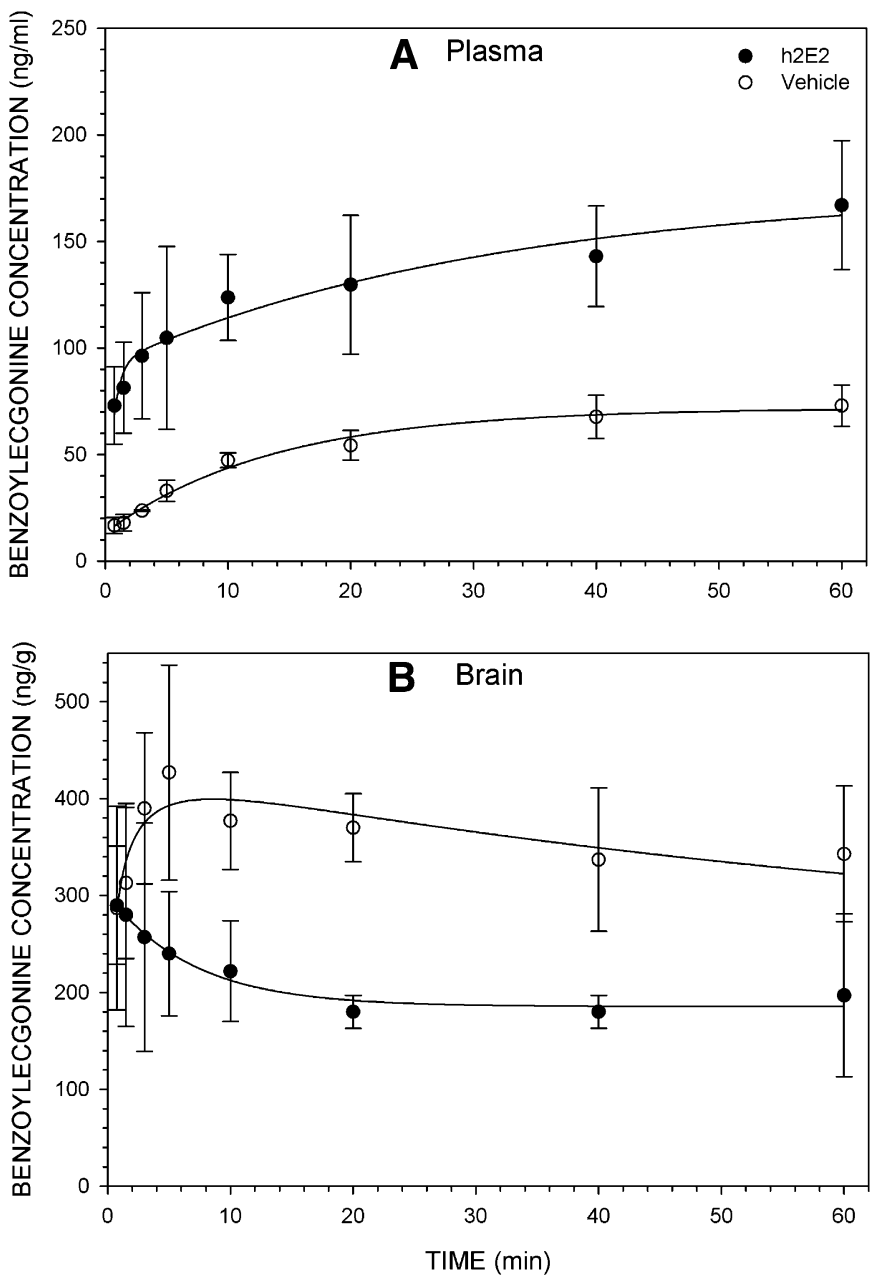

Fig. 4. The effect of $\mathrm{h} 2 \mathrm{E} 2$ on the formation of $\mathrm{BE}$ via cocaine metabolism in plasma (A) and brain (B) in rats. Concomitant to determination of cocaine concentrations using GC/MS, the concentrations of BE were determined. Each data point represents the mean \pm S.E.M. from three rats. In the absence of h2E2 (open circles), BE is formed at a relatively constant rate in plasma (A), but in the brain (B) it is present in higher concentrations that decline over time. In contrast, the presence of h2E2 (closed circles) causes an increase in BE levels in plasma (A) and in the brain (B) a corresponding decrease in BE formation. 
$\left(\mathrm{t}_{1 / 2 \beta}\right)$ value for h2E2 was relatively long and similar to that reported for other human antibodies in rats (Bazin-Redureau et al., 1997) and indicates that h2E2's effects in humans will potentially be long lasting. The initial more rapid decrease of plasma h2E2 in rats most likely corresponds to the distribution of h2E2 from plasma to the interstitial spaces, and this is typical of human $\operatorname{IgG1}$ antibodies in rodents.

In this study, we report for the first time the effects of h2E2 on cocaine pharmacokinetics in rats. The effect of h2E2 on cocaine pharmacokinetics is equivalent to an increase in plasma protein binding for cocaine. This results in the observed dramatic reduction in the total plasma clearance of cocaine, although the intrinsic clearance of cocaine may not be reduced to the same extent as for total plasma clearance. An initial distribution phase for cocaine was observed in the presence of h2E2, although the process was much slower and less pronounced than the distribution phase observed in the absence of $\mathrm{h} 2 \mathrm{E} 2$. Although this initial decrease in plasma cocaine concentrations in the presence of h2E2 is assumed to represent the distribution of cocaine from plasma to other tissues, it was not distributing to the brain. Therefore, the pharmacokinetics of cocaine in the presence of $\mathrm{h} 2 \mathrm{E} 2$ may be more complex in rats than that of mice, in which no initial distribution phase was observed (in the presence of 2E2) (Norman et al., 2007), but the efficacy of h2E2 as defined as the prevention of cocaine entry into the brain was similar. The ability of recombinant h2E2 to inhibit cocaine entry into the brain in rats observed in this study is consistent with previous reports in rats that active immunization-induced anti-cocaine antibodies decreased cocaine levels in rat brain after i.v., intranasal, or i.p. cocaine administration (Fox et al., 1996; Fox, 1997; Carrera et al., 2000). The ability of h2E2 to decrease brain cocaine concentrations is also consistent with the previous reports of mAb-induced reductions in the brain concentrations of other psychoactive drugs such as phencyclidine (Valentine and Owens, 1996; Proksch et al., 2000), methamphetamine (Laurenzana et al., 2003), and nicotine (Keyler et al., 2005).

Consistent with previous reports (Booze et al., 1997; Warner and Norman, 2000), BE was the major metabolite of cocaine in rats. Indeed, the concentrations of EME in both plasma and brain were negligible under the conditions of this in vivo study. In contrast, in mice, EME was the major metabolite (Norman et al., 2007). Despite the higher affinity of h2E2 for BE relative to that for EME, there appeared to be no attenuation of the magnitude of the effects of h2E2 on decreasing the brain concentrations of cocaine and increasing plasma concentrations in rats. Thus, the in vivo efficacy of h2E2 was independent of the route of cocaine metabolism. It should be noted that the current studies were conducted only after a single cocaine injection, and, after repeated cocaine injections that result in higher BE concentrations, there may be some loss of h2E2 efficacy. However, when cocaine was self-administered by rats over several hours, which presumably results in higher BE concentrations than those resulting from a single low-dose cocaine injection, the effects of the anticocaine mAb 2E2 were stable (Norman et al., 2009). In humans, BE and EME are both produced by the metabolism of cocaine at ratios that are between those of rats and mice, and so there would be no expectation that the clinical efficacy of h2E2 should be diminished in the presence of differing routes of cocaine metabolism.

The finding of higher concentrations of $\mathrm{BE}$ in the brain relative to those in plasma in the control rats suggests that at least some of the cocaine that enters the brain is metabolized there to BE, which is consistent with previous reports in animals and humans (Karch, 1996). As the brain cocaine concentration rapidly declined due to cocaine's metabolism and redistribution to plasma, this resulted in no further increase in brain BE concentrations. The lower brain BE concentrations in the presence of h2E2 (in the plasma) reflect the lowered brain cocaine concentration, which is also consistent with the idea that cocaine is metabolized to $\mathrm{BE}$ in the brain. The relatively small decrease in brain $\mathrm{BE}$ concentrations in the presence of plasma h2E2 relative to the dramatic $\mathrm{h} 2 \mathrm{E} 2$-indued reductions in brain cocaine concentrations may indicate that $\mathrm{BE}$ can enter the brain from the periphery. However, it has been reported that $\mathrm{BE}$ does not cross the blood-brain barrier (Misra et al., 1975) due to its physicochemical properties. Further studies on the formation and/or transport of BE across the blood-brain barrier will be required to fully understand the implications of these findings.

The increased plasma BE concentrations in the presence of h2E2 are consistent with cocaine being sequestered and then available to be metabolized to BE in the peripheral circulation. Curiously, despite the greater than 16-fold increase in peak plasma cocaine concentrations in the presence of h2E2, plasma BE concentrations were increased less than threefold. Whether the metabolism of cocaine to BE in the presence of h2E2 is reduced or whether there is a mAb-induced alteration in the routes of cocaine elimination from the periphery that results in altered BE levels will require further studies. However, there was no evidence of an increase in EME formation.

In summary, the high-affinity anti-cocaine $\mathrm{mAb}$ h2E2 retained the binding properties of $2 \mathrm{E} 2$ and in rats limited the distribution of cocaine to the plasma, thus decreasing the levels of cocaine reaching the brain without inhibiting the elimination of cocaine. In rats in which cocaine is metabolized predominantly to $\mathrm{BE}$, as opposed to mice in which cocaine is metabolized predominantly to EME, the efficacy of h2E2 (defined as the ability to decrease brain cocaine concentrations) is similar. Therefore, the efficacy of h2E2 should be independent of species, animal size, or route of cocaine metabolism. These findings further support the general concept of the usefulness of immunotherapy for the treatment of drug abuse and are consistent with $\mathrm{mAb}$ h2E2 being a lead candidate for development as a passive immunotherapy for cocaine abuse.

\section{Acknowledgments}

The authors thank Ernie Chaffin, Robert Topmiller, and Katie Gabbard from the Hamilton County Coroner's Office (Cincinnati, OH) for conducting the analytical quantitation of cocaine and metabolite concentrations. The authors thank Catalent for re-engineering $2 \mathrm{E} 2$ to h2E2 and for manufacturing the recombinant h2E2 used in these studies. The authors also thank Purabi Dey for technical assistance, Erin Bartley and Hanna Dasenbrock for assistance with collecting blood samples from rats, and Dr. Vladimir L. Tsibulsky for helpful discussions.

\section{Authorship Contributions}

Participated in research design: Norman, Ball.

Conducted experiments: Norman, Gooden, Tabet.

Performed data analysis: Norman, Gooden, Tabet.

Wrote or contributed to the writing of the manuscript: Norman, Gooden, Tabet, Ball.

\section{References}

Bazin-Redureau MI, Renard CB, and Scherrmann JM (1997) Pharmacokinetics of heterologous and homologous immunoglobulin $\mathrm{G}, \mathrm{F}\left(\mathrm{ab}^{\prime}\right)_{2}$ and $\mathrm{Fab}$ after intravenous administration in the rat. J Pharm Pharmacol 49:277-281.

Booze RM, Lehner AF, Wallace DR, Welch MA, and Mactutus CF (1997) Dose-response cocaine pharmacokinetics and metabolite profile following intravenous administration and arterial sampling in unanesthetized, freely moving male rats. Neurotoxicol Teratol 19:7-15.

Bleck GT (2012) Consistent production of genetically stable mammalian cell lines. BioPharm International 25:56-59.

Carrera MR, Ashley JA, Parsons LH, Wirsching P, Koob GF, and Janda KD (1995) Suppression of psychoactive effects of cocaine by active immunization. Nature 378:727-730.

Carrera MR, Ashley JA, Zhou B, Wirsching P, Koob GF, and Janda KD (2000) Cocaine vaccines: antibody protection against relapse in a rat model. Proc Natl Acad Sci USA 97:6202-6206.

Choudhri TF, Hoh BL, Solomon RA, Connolly ES, Jr, and Pinsky DJ (1997) Use of a spectrophotometric hemoglobin assay to objectively quantify intracerebral hemorrhage in mice. Stroke 28:2296-2302.

Fox BS (1997) Development of a therapeutic vaccine for the treatment of cocaine addiction. Drug Alcohol Depend 48:153-158. 
Fox BS, Kantak KM, Edwards MA, Black KM, Bollinger BK, Botka AJ, French TL, Thompson TL, Schad VC, and Greenstein JL, et al. (1996) Efficacy of a therapeutic cocaine vaccine in rodent models. Nat Med 2:1129-1132.

Karch SB (1996) The Pathology of Drug Abuse, 2nd ed, CRC Press, Boca Raton, FL.

Keyler DE, Roiko SA, Benlhabib E, LeSage MG, St Peter JV, Stewart S, Fuller S, Le CT, and Pentel PR (2005) Monoclonal nicotine-specific antibodies reduce nicotine distribution to brain in rats: dose- and affinity-response relationships. Drug Metab Dispos 33:10561061

Kosten TR, Rosen M, Bond J, Settles M, Roberts JS, Shields J, Jack L, and Fox B (2002) Human therapeutic cocaine vaccine: safety and immunogenicity. Vaccine 20:1196-1204.

Kosten TR and Owens SM (2005) Immunotherapy for the treatment of drug abuse. Pharmacol Ther 108:76-85.

Laurenzana EM, Byrnes-Blake KA, Milesi-Hallé A, Gentry WB, Williams DK, and Owens SM (2003) Use of anti-(+)-methamphetamine monoclonal antibody to significantly alter (+)-methamphetamine and (+)-amphetamine disposition in rats. Drug Metab Dispos 31 $1320-1326$.

Lonberg N (2005) Human antibodies from transgenic animals. Nat Biotechnol 23:1117-1125.

Martell BA, Mitchell E, Poling J, Gonsai K, and Kosten TR (2005) Vaccine pharmacotherapy for the treatment of cocaine dependence. Biol Psychiatry 58:158-164.

Mets B, Winger G, Cabrera C, Seo S, Jamdar S, Yang G, Zhao K, Briscoe RJ, Almonte R, and Woods JH, et al. (1998) A catalytic antibody against cocaine prevents cocaine's reinforcing and toxic effects in rats. Proc Natl Acad Sci USA 95:10176-10181.

Misra AL, Nayak PK, Bloch R, and Mulé SJ (1975) Estimation and disposition of [3H]benzoylecgonine and pharmacological activity of some cocaine metabolites. J Pharm Pharmaco 27:784-786.

Norman AB, Tabet MR, Norman MK, Buesing WR, Pesce AJ, and Ball WJ (2007) A chimeric human/murine anticocaine monoclonal antibody inhibits the distribution of cocaine to the brain in mice. $J$ Pharmacol Exp Ther 320:145-153.
Norman AB, Norman MK, Buesing WR, Tabet MR, Tsibulsky VL, and Ball WJ (2009) The effect of a chimeric human/murine anti-cocaine monoclonal antibody on cocaine self-administration in rats. $J$ Pharmacol Exp Ther 328:873-881.

Norman AB and Ball WJ, Jr (2012) Predicting the clinical efficacy and potential adverse effects of a humanized anticocaine monoclonal antibody. Immunotherapy 4:335-343.

Paula S, Tabet MR, Farr CD, Norman AB, and Ball WJ, Jr (2004) Three-dimensional quantitative structure-activity relationship modeling of cocaine binding by a novel human monoclonal antibody. J Med Chem 47:133-142.

Proksch JW, Gentry WB, and Owens SM (2000) Anti-phencyclidine monoclonal antibodies provide long-term reductions in brain phencyclidine concentrations during chronic phencyclidine administration in rats. J Pharmacol Exp Ther 292:831-837.

Redwan RM, Larsen NA, Zhou B, Wirsching P, Janda KD, and Wilson IA (2003) Expression and characterization of a humanized cocaine-binding antibody. Biotechnol Bioeng 82:612-618.

Valentine JL and Owens SM (1996) Antiphencyclidine monoclonal antibody therapy significantly changes phencyclidine concentrations in brain and other tissues in rats. J Pharmacol Exp Ther 278:717-724.

Warner A and Norman AB (2000) Mechanisms of cocaine hydrolysis and metabolism in vitro and in vivo: a clarification. Ther Drug Monit 22:266-270.

Zhang Y, Huo M, Zhou J, and Xie S (2010) PKSolver: an add-in program for pharmacokinetic and pharmacodynamic data analysis in Microsoft Excel. Comput Methods Programs Biomed 99:306-314.

Address correspondence to: Dr. Andrew B. Norman, Department of Pharmacology and Cell Biophysics, University of Cincinnati, College of Medicine, 231 Albert Sabin Way, Cincinnati, OH 45267-0575. E-mail: andrew.norman@uc.edu 